R. Campanilho-Marques ${ }^{1,2}$, I. Cordeiro ${ }^{1,2} .{ }^{1}$ Hospital de Santa Maria, Centro Hospitalar Universitário de Lisboa Norte, Centro Académico de Medicina de Lisboa, Lisbon, Portugal, Serviço de Reumatologia e Doenças Ósseas Metabólicas, Lisbon, Portugal; ${ }^{2}$ Instituto de Medicina Molecular, Faculdade de Medicina, Universidade de Lisboa, Centro Académico de Medicina de Lisboa, Lisboa, Portugal, Lisbon, Portugal, Unidade de Investigação em Reumatologia, Lisbon, Portugal; ${ }^{3}$ Centro Hospitalar Vila Nova de Gaia/Espinho, Serviço de Reumatologia, Vila Nova de Gaia, Portugal; ${ }^{4}$ Centro Hospitalar e Universitário de Coimbra, Coimbra, Portugal, Serviço de Reumatologia, Coimbra, Portugal; ${ }^{5}$ Hospital Garcia de Orta, Almada, Portugal, Serviço de Reumatologia, Almada, Portugal; ${ }^{6}$ Centro Hospitalar Lisboa Ocidental, Hospital Egas Moniz, Lisboa, Portugal, Serviço de Reumatologia, Lisbon, Portugal; ${ }^{7}$ Centro Hospitalar e Universitário São João, Porto, Portugal, Serviço de Reumatologia, Porto, Portugal; ${ }^{8}$ Serviço de Reumatologia, Hospital de Braga, Braga, Portugal; ${ }^{9}$ Serviço de Reumatologia, Unidade Local de Saúde Do Alto Minho, Ponte de Lima, Portugal, Ponte de Lima, Portugal; ${ }^{10}$ Centro Hospitalar Universitário do Algarve, Faro, Portugal, Serviço de Reumatologia, Faro, Portugal

Background: Antisynthetase syndrome (ASyS) may have different clinical phenotypes and outcomes associated with different anti-aminoacyl RNA-synthetase (anti-ARS) antibodies. Its wide clinical spectrum can include inflammatory myopathy, interstitial lung disease (ILD), arthritis, fever, mechanic's hands, and Raynaud phenomenon (RP). Objectives: To describe a nationwide, multicentre cohort of Portuguese patients with ASyS. Methods: Retrospective analysis of patients with ASyS from nine Portuguese Rheumatology centers. Data on patients' signs and symptoms, laboratory results, pulmonary radiological findings (computed tomography) and treatment (immunomodulators) were collected.

Results: Among the 70 patients included, 42 patients (60\%) were anti-Jo1-positive, 11 (15.7\%) were anti-PL12-positive, $10(14.3 \%)$ were anti-PL7-positive, 4 (5.7\%) were anti-EJ-positive and $2(2.9 \%)$ were anti-OJ positive. In one patient it was not possible to identify the type of antibody. Antibody overlap was found in 15 patients (21.4\%), who were positive for anti-Ro52 antibodies. The general clinical characteristics are shown in Table 1. The diagnostic delay was greater in patients positive for anti-OJ, followed by anti-Jo-1 and anti-PL12. The follow-up was shorter for anti-PL7 and anti-OJ-positive patients. Anti-PL7-positive patients had lower rates of arthritis when compared to anti-Jo1 $(p<0.01)$. When compared with anti-Jo-1 ARS, myositis was less common in anti-PL12 $(p<0.01)$. ILD prevalence was similar in the different ARS subgroups. Glucocorticoids (GCs) were the most frequently used class of drugs. A more conservative treatment plan (e.g. GCs plus methotrexate or azathioprine) was the treatment of choice in ASyS with myositis and/or arthritis involvement. Rituximab or mycophenolate mofetil were preferred when lung involvement occurred. Only two deaths were reported, being one associated with lung neoplasia.

Conclusion: This is the first study investigating the clinical phenotypes of Portuguese patients with ASyS. These results are generally concordant with data retrieved from international cohorts.

REFERENCES:

[1] Mahler M, Miller FW, Fritzler MJ. Idiopathic inflammatory myopathies and the anti-synthetase syndrome: a comprehensive review. Autoimmun Rev 2014;13:367-71.
Disclosure of Interests: None declared

DOI: 10.1136/annrheumdis-2021-eular.3068

\section{POS0871 CHARACTERISTICS OF INTERSTITIAL LUNG DISEASE IN PATIENTS WITH SYSTEMIC SCLEROSIS DURING LONG TERM FOLLOW-UP, SINGLE CENTER EXPERIENCE}

S. Keret ${ }^{1}$, Y. Braun-Moscovici ${ }^{2}$, M. Yigla ${ }^{3}$, V. Shataylo ${ }^{4}$, L. Guralnik ${ }^{5}$, A. BalbirGurman ${ }^{2} .{ }^{1}$ Rambam Health Care Campus, Internal Medicine C, Haifa, Israel; ${ }^{2}$ Rambam Health Care Campus, Rheumatology Institute, Rappaport Faculty of Medicine, Technion, Haifa, Israel; ${ }^{3}$ Rambam Health Care Campus, Pulmonary Institute, Haifa, Israel; ${ }^{4}$ Rambam Health Care Campus, Rheumatology Institute, Haifa, Israel; ${ }^{5}$ Rambam Health Care Campus, Imaging Department, Rappaport Faculty of Medicine, Technion, Haifa, Israel

Background: ILD is one the leading causes of morbidity and mortality in patients with SSc. Diagnosis of SScILD is based on signs of fibrosis on chest $x$-rays or HRCT. Particular measurement of lung volumes by FVC and in gas exchange by DLCO supports the diagnosis. Associations between clinically significant SSc ILD and male gender, age, DcSSc, topoisomerase antibodies, low FVC at baseline, widespread lung involvement on baseline HRCT, and higher decline rate of FVC and DLCO during followup were reported. A standardized approach to assessing and treating SSc and SSCILD in particular have been proposed. Main treatment regimens include cyclophosphamide and mycophenolate mofetil; recently antifibrotic drug nintedanib showed significant efficacy in hindering FVC decline rate in patients with SSc ILD. The data on survival changes in SSc generally and SScILD are conflicting.

Objectives: To analyze demographic and clinical features and mortality of patients with SSc ILD.

Methods: A retrospective study on Rambam Health Care Campus prospective cohort of SSc patients fulfilled ACR and EULAR Classification Criteria 2013 for the period between January 2000 and September 2020 was performed. Patients were recruited at one of their early visits to the clinic. The majority of recruited patients were included into EUSTAR prospective cohort 042, since 2004 the Rheumatology Institute at Rambam is affiliated to EUSTAR registry project. Data on patients not registered in EUSTAR database but treated at our institution was extracted via hospital electronic records. Patients with lung involvement underwent baseline and annual HRCT and pumonary function tests in addition to clinical assessment during their visit to combined rheumatology-pulmonology clinic. We registered age, gender, ethnicity, date of SSc diagnosis and ILD diagnosis, disease duration, SSc subset, pulmonary, cardiac, renal, and muscle involvement, treatment used, autoantibodies, FVC, DLCO, HRCT and pulmonary artery pressure. Statistical analysis included t-test, Pearson's Chi-squared test, Fisher's test, and Cox Regression analysis with $p$ value less than 0.05 as significant.

Results: Among 446 SSc patients (female $82.3 \%$, mean age 46.5 and disease duration 11.6 years, DcSSc $39.2 \% ; 27.4 \%$ dead during follow-up) 141 patients had ILD. Comparison between patient with ILD and witout ILD showed significant differences in term of nationality (Arabs $34 \%$ vs $18.7 \%$ ), SSc related death $78.3 \%$ vs $50.7 \%)$, DcSSc ( $68.8 \%$ vs $25.6 \%)$, topoisomerase antibodies

Table 1. Patient characteristics according to the anti-ARS. ILD - interstitial lung disease; IQR- interquartile range; NSIP - Non-specific interstitial pneumonia; UIP - Usual interstitial pneumonia; yrs - years

\begin{tabular}{|c|c|c|c|c|c|c|}
\hline Variables & Overall, $\mathrm{n}=70$ & $\begin{array}{l}\text { Jo- } 1, n=42 \\
(60 \%)\end{array}$ & $\mathrm{PL}-12, \mathrm{n}=11$ (15.7\%) & PL-7, n=10 (14.3\%) & $E J, n=4(5.7 \%)$ & OJ, $n=2(2.9 \%)$ \\
\hline Mean age at onset, yrs & $52 \pm 15$ & $46.6 \pm 14.4$ & $55.2 \pm 14.7$ & $56.5 \pm 12.5$ & $56.3 \pm 11.2$ & $73.5 \pm 2.1$ \\
\hline Female, $\mathrm{n}(\%)$ & $49(70)$ & $29(69)$ & $9(81.8)$ & $7(70)$ & $2(50)$ & $2(100)$ \\
\hline Median age in years at disease onset (IQR) & $52(15-75)$ & $48(15-70)$ & $59(20-70)$ & $62(39-73)$ & $60(40-65)$ & $73.5(72-75)$ \\
\hline Median follow-up time in yrs (IQR) & $3(0-32)$ & $5(0-32)$ & $3(0-13)$ & $1(1-4)$ & $4(2-21)$ & $1(0-2)$ \\
\hline Median diagnostic delay in yrs (IQR) & $6(1-33)$ & $7(1-33)$ & $7(2-19)$ & $4(1-23)$ & $1.5(1-2)$ & $12.5(2-21)$ \\
\hline \multirow[t]{2}{*}{ Myositis, n (\%) and Comparison Anti-Jo.1 ARS vs PL-12 and PL-7 } & $36(51.4)$ & $25(59.5)$ & $3(27.3)$ & $4(40)$ & $3(75)$ & 0 \\
\hline & & & ${ }^{*} \mathrm{p}<0.01$ & $\mathrm{p}=0.7$ & - & - \\
\hline \multirow[t]{2}{*}{ ILD, n (\%) and Comparison Anti-Jo.1 ARS vs PL-12 and PL-7 } & $53(75.7)$ & $33(78.6)$ & $8(72.7)$ & $6(60) p=0.56$ & $4(100)$ & $1(50)$ \\
\hline & & & $p=0.98$ & & - & - \\
\hline ILD pattern - NSIP, n (\%) & $30(56.6)$ & $18(54.5)$ & $6(75)$ & $3(50)$ & $1(25)$ & 0 \\
\hline ILD pattern - UIP, $\mathrm{n}(\%)$ & $6(11.3)$ & $3(9.1)$ & $1(12.5)$ & $1(16.7)$ & $1(25)$ & 0 \\
\hline ILD pattern - other specific pattern, $n(\%)$ & $6(11.3)$ & 4 (12.1) & 0 & 2 (33.3) & $1(25)$ & 0 \\
\hline ILD pattern - non-specific pattern, $\mathrm{n}(\%)$ & $11(15.7)$ & $8(24.2)$ & $1(12.5)$ & 0 & $1(25)$ & $1(100)$ \\
\hline Mechanic's hands (\%), $\mathrm{n}(\%)$ & $23(32.9)$ & $14(33.3)$ & $3(27.3)$ & $2(20)$ & 0 & $1(50)$ \\
\hline General impairment, $n(\%)$ & $18(25.7)$ & $11(26.2)$ & $3(27.3)$ & $2(20)$ & $2(50)$ & 0 \\
\hline Fever, $\mathrm{n}(\%)$ & $7(10)$ & $4(9.5)$ & $2(20.2)$ & 0 & $1(25)$ & 0 \\
\hline Raynaud phenomenon, $\mathrm{n}(\%)$ & $22(31.4)$ & $11(26.2)$ & $7(63.6)$ & 0 & $2(50)$ & 0 \\
\hline \multirow[t]{2}{*}{ Arthritis, $\mathrm{n}(\%)$ and Comparison Anti-Jo.1 ARS vs PL-12 and PL-7 } & $43(61.4)$ & $29(69)$ & $5(45.4)$ & $2(20)$ & $2(50)-$ & $1(50)-$ \\
\hline & & & $\mathrm{p}=0.07$ & ${ }^{*} \mathrm{p}<0.01$ & & \\
\hline Malignancy, n (\%) & $4(5.7)$ & $3(7.1)$ & $1(9.1)$ & 0 & 0 & 0 \\
\hline Deaths, n (\%) & $2(2.9)$ & $2(2.4)$ & 0 & 0 & 0 & $1(50)$ \\
\hline
\end{tabular}


(61.7\% vs $24.9 \%)$, myopathy $(21.3 \%$ vs $10.2 \%)$ and pulmonary hypertension $(34.8 \%$ vs $22.3 \%)$. Significantly more SSc ILD patients were treated with cyclophosphamide, mycophenolate mofetil and azathioprine. Survival Kaplan-Meier curve patiets demonstrate reduced survival in patients with ILD $(p<0.01)$. Five years survival rates between years 2000 and 2015 have not changed significant. Mortality risk assessed with Cox regression analysis in the whole group was significantly higher in males, Arabs, DcSSc, elder age, heart and muscle involvement, and treatment with CYC. In the ILD group, the mortality was significantly higher in Arabs (3.3 times), elder age (8.9 times), presence of PAH (3.1 times) and treatment with CYC (2.8 times) compared to patients without ILD.

Conclusion: In our SSc cohort, ILD affected about third of patients and had major impact on patients' outcome. Male gender, Arab nationality, elder age, DcSSc, topoisomerase antibodies, heart and muscle involvement were significantly associated with worst prognosis. Despite active approach to assessing and treatment, survival rates of patients with SSc and SSc-ILD have not improved in last decades. Enrichment of the cohort with severe patients to a tertiary center due to reference bias and low efficacy of existed immunomodulatory drugs in SSc and in SSc related ILD particularly, could explain our results.

Disclosure of Interests: None declared

DOI: 10.1136/annrheumdis-2021-eular.3105

\section{POS0872 CLINICAL FEATURES AND OUTCOME OF 1054 PATIENTS WITH SYSTEMIC SCLEROSIS: AN ANALYSIS OF THE PORTUGUESE REUMA.PT REGISTRY FOR SCLERODERMA (REUMA.PT/SSC)}

R. Freitas ${ }^{1}$, P. Martins ${ }^{2}$, E. Dourado ${ }^{2}$, M. J. Salvador ${ }^{3,4}$, T. Santiago $^{3,4}$, I. Cordeiro' ${ }^{2}$, B. M. Fernandes ${ }^{5}$, F. Guimarães ${ }^{6}$, S. Garcia ${ }^{5}$, B. Samões ${ }^{7}$, N. Gonçalves ${ }^{8}$, M. H. Fernandes Lourenco ${ }^{8}$, A. S. Pinto ${ }^{9}$, M. Rocha ${ }^{10}$, M. Couto ${ }^{11}$, E. Costa ${ }^{12}$, F. Araújo ${ }^{13}$, C. Resende ${ }^{2}$, F. Godinho ${ }^{1}$, A. Cordeiro ${ }^{1}$, M. J. Santos ${ }^{1,14} .{ }^{1}$ Hospital Garcia de Orta, Rheumatology Department, Almada, Portugal; ${ }^{2}$ Centro Hospitalar Lisboa Norte, Rheumatology Department, Lisboa, Portugal; ${ }^{3}$ Centro Hospitalar e Universitário de Coimbra, Rheumatology Department, Coimbra, Portugal; ${ }^{4}$ University of Coimbra, Rheumatology Department, Coimbra, Portugal; ${ }^{5}$ São João Universitary Hospital Center, Rheumatology Department, Porto, Portugal; ${ }^{6}$ Centro Hospitalar do Alto Minho, Rheumatology Department, Ponte de Lima, Portugal; ${ }^{7}$ Centro Hospitalar Vila Nova de Gaia, Rheumatology Department, Vila Nova de Gaia, Portugal; ${ }^{8}$ Centro Hospitalar Lisboa Ocidental, Rheumatology Department, Lisboa, Portugal; ${ }^{9}$ Unidade de Saúde Local da Guarda, Rheumatology Department, Guarda, Portugal; ${ }^{10}$ Centro Hospitalar Universitário do Algarve, Rheumatology Department, Faro, Portugal; ${ }^{11}$ Centro Hospitalar Tondela - Viseu, Rheumatology Department, Viseu, Portugal; ${ }^{12}$ Hospital de Braga, Rheumatology Department, Braga, Portugal; ${ }^{13}$ Hospital de Sant'Ana, Rheumatology Department, Parede, Portugal; ${ }^{14}$ Lisbon University, Medical School, Lisboa, Portugal

Background: Systemic sclerosis (SSc) may present distinctive manifestations and survival in different ethnic and geographic groups.

Objectives: To describe the clinical features, treatments, and survival of adult SSc patients registered in Reuma.pt/SSc.

Methods: Demographic features, SSc subsets, fulfilment of classification criteria, clinical and immunologic characteristics, comorbidities, medication and deaths were reviewed. Survival was calculated for patients included in the registry within the first 2 years of diagnosis.

Results: In total, 1054 patients were included, $87.5 \%$ female, mean age at diagnosis $52.7 \pm 14.8$ years. The most common subset was limited cutaneous (Ic)SSc (56.3\%), followed by diffuse cutaneous (dc)SSc (17.5\%), preclinical SSc $(13 \%)$, overlap syndrome $(9.8 \%)$ and SSc sine scleroderma $(3.3 \%)$. Raynaud's phenomenon $(93.4 \%)$ and skin thickening $(76.9 \%)$ were the most observed manifestations. Gastrointestinal $(62.8 \%$ vs $47.8 \%)$, pulmonary $(59.5 \%$ vs $23 \%)$ and cardiac (12.8\% vs $6.9 \%)$ involvement were significantly more prevalent in dcSSc compared to IcSSc (Table 1). $52.5 \%$ of patients were ACA positive and $21 \%$ anti-topoisomerase positive, with significant differences between IcSSc and dcSSc. One third of patients was treated with immunomodulators, $53.6 \%$ with vasodilators, $23 \%$ received glucocorticoids and $2.3 \%$ biologics.

During the median follow-up 12.4 years, 83 deaths $(7.9 \%)$ were verified. The overall 1,2 and 5 years survival was $98.0 \%, 96.8 \%$ and $92.6 \%$ respectively, without significant differences between IcSSc and dcSSc (Figure 1).

Conclusion: Reuma.pt/SSc register is useful in routine patient monitoring and contributes to improve knowledge about this rare and complex disease. Clinical features of Portuguese SSc patients are similar to what has been described in other populations although the overall 5 -year survival in recently diagnosed patients appears to be higher than previously reported.
Table 1. Cumulative clinical and immunologic characteristics of Portuguese SSc patients

\begin{tabular}{|c|c|c|c|c|}
\hline & & $\begin{array}{l}\text { Limited cuta- } \\
\text { neous SSc }\end{array}$ & $\begin{array}{l}\text { Diffuse cuta- } \\
\text { neous SSc }\end{array}$ & \\
\hline Clinical and immunologic features & $\mathrm{N}=1054$ & & & \\
\hline Skin involvement $-\mathrm{N}(\%) \mathrm{N}=987$ & \multicolumn{2}{|c|}{$688(90.6) 525(90.7)$} & $180(100)$ & $<0.01$ \\
\hline Skin thickening * $-\mathrm{N}(\%) \mathrm{N}=962$ & \multicolumn{2}{|c|}{680 (76.9)512 (88.9) } & $180(100)$ & $<0.01$ \\
\hline Digital ulcers $-\mathrm{N}(\%) \mathrm{N}=970$ & \multicolumn{2}{|c|}{$325(33.5) 186(34.7)$} & $4(51.5)$ & $<0.01$ \\
\hline Raynaud's Phenomenon - N (\%) N=1010 & \multirow{2}{*}{\multicolumn{2}{|c|}{$\begin{array}{l}943(93.4) 539(95.7) \\
346(45.6) 247(42.7)\end{array}$}} & $157(92.4)$ & 0.06 \\
\hline Musculoskeletal involvement $-\mathrm{N}(\%)$ & & & $99(55)$ & $<0.01$ \\
\hline \multicolumn{5}{|l|}{$\mathrm{N}=972$} \\
\hline Cardiac involvement $-\mathrm{N}(\%)-\mathrm{N}=924$ & $71(7.7)$ & $36(6.9)$ & $19(12.8)$ & 0.02 \\
\hline Renal involvement $-\mathrm{N}(\%)-\mathrm{N}=917$ & $17(1.9)$ & $8 ! 1.5)$ & $6(4.1)$ & 0.07 \\
\hline \multicolumn{3}{|c|}{ Gastrointestinal involvement - N(\%) N=933508 (48.2)277 (47.8) } & $113(62.8)$ & $<0.01$ \\
\hline Pulmonary involvement $-\mathrm{N}(\%) \mathrm{N}=915$ & \multicolumn{2}{|c|}{$261(28.5) 119(23)$} & $88(59.5)$ & $<0.01$ \\
\hline PAH $-N(\%) N=871$ & $14(1.6)$ & $10(2)$ & $1(0.7)$ & 0.23 \\
\hline Intersticial lung disease $-\mathrm{N}(\%) \mathrm{N}=765$ & \multicolumn{2}{|c|}{$218(28.5) 100(22.7)$} & $75(57.7)$ & $<0.01$ \\
\hline Antinuclear antibodies $-\mathrm{N}(\%) \mathrm{N}=1040$ & \multirow{2}{*}{\multicolumn{2}{|c|}{$\begin{array}{l}934(89.8) 522(90.2) \\
540(52.6) 383(67.1)\end{array}$}} & $154(88.5)$ & 0.57 \\
\hline Anti-centromere $-\mathrm{N}(\%) \mathrm{N}=1027$ & & & $16(9.5)$ & $<0.01$ \\
\hline Anti-Scl70 - N(\%) N=1020 & $214(21)$ & $12(3.3)$ & $104(60.1)$ & $<0.01$ \\
\hline $\begin{array}{l}\text { Anti-RNA polymerase III - N(\%) N=710 } \\
\text { Comorbidities }\end{array}$ & $25(3.5)$ & $12(3.3)$ & $7(5.6)$ & 0.38 \\
\hline Hypertension $-\mathrm{N}(\%) \mathrm{N}=431$ & $117(27.1)$ & $76(29.7)$ & $67(20.7)$ & 0.1 \\
\hline Hyperlipidemia $-\mathrm{N}(\%) \mathrm{N}=431$ & $71(13.4)$ & $72(12.2)$ & $24(15.9)$ & 0.08 \\
\hline Neoplasia - N(\%) N=1054 & $29(2.8)$ & $12(2.1)$ & $7(3.9)$ & 0.14 \\
\hline
\end{tabular}

PDE-5 (phosdiasterase-5); PPIs (proton pump inhibitors); PAH-Pulmonary arterial hypertension confirmed by right heart catheterization. Immunomodulators includes Metothrexate Leflunomide, Hydroxycloroquine; Azathioprine, Mycophenolate Mofetil and Cyclophosphamide; ${ }^{*}$ Does not include sclerodactyly.

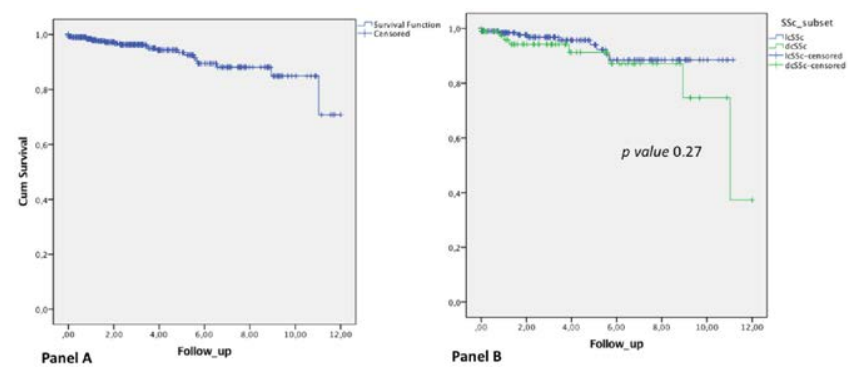

Figure 1. Panel A - Survival in years from diagnosis of patients with SSc included in Reuma $\mathrm{pt}$ in the first 2 years of disease ( $\mathrm{N}=472$ ). Panel B - survival according to SSc subset (IcSSc and dcSSC).

Disclosure of Interests: None declared DOI: 10.1136/annrheumdis-2021-eular.3159

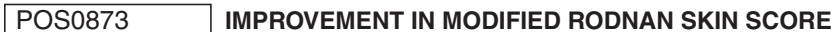 (MRSS) IN SYSTEMIC SCLEROSIS PATIENTS TREATED WITH RITUXIMAB: A SYSTEMATIC REVIEW AND META-ANALYSIS OF THE SCIENTIFIC LITERATURE}

J. L. Puga Guzmán ${ }^{1}$, D. Fernández Fernández ${ }^{2,3}$, R. Dos-Santos ${ }^{2,3}$, I. González Fernández ${ }^{2,3}$, E. Perez-Pampín ${ }^{3,4}$, A. Souto Vilas ${ }^{3,4}$, A. Mera Varela ${ }^{3,4}$ ${ }^{1}$ Facultade de Medicina e Odontoloxía - USC, Medicine, Santiago de Compostela, Spain; ${ }^{2}$ Hospital Clínico Universitario de Santiago, Rheumatology, Santiago de Compostela, Spain; ${ }^{3}$ University of Santiago de Compostela, Medicine, Santiago de Compostela, Spain; ${ }^{2}$ Hospital Clínico Universitario de Santiago, Rheumatology, Santiago de Compostela, Spain

Background: Systemic sclerosis (SSc) is a chronic autoimmune disease of the connective tissue characterized by vascular disease and fibrosis in different organs and systems such as lung and skin (1). Recently, several case reports and small series of patients reported on the efficacy of rituximab in SSc, showing a possible improvement in skin and lung affectations (2). However, registered clinical trials are lacking to determine factors associated with response, maintenance regimen, and long-term efficacy of rituximab in SSc.

Objectives: To analyze the efficacy of Rituximab in the treatment of skin fibrosis using the changes of the modified Rodnan Skin Score (mRSS) of patients diagnosed with systemic sclerosis from the data published in Registered Clinical Trials (RCTs) in the scientific literature.

Methods: We perform a systematic review and a meta-analysis using the main electronic databases to locate all the articles available so far: Medline, Embase, Cochrane Library and Web of science and ACR and EULAR abstracts congress were extracted to assess efficacy outcomes. That efficacy was measured based on the variation of mRSS at 12,24 and 48 weeks for patients treated with Rituximab versus patients treated with another drug or placebo. 